\title{
The Role of Magnetic Topology in the Heating of Active Region Coronal Loops
}

\author{
J.-Y. Lee ${ }^{1,2}$, Graham Barnes ${ }^{2}$, K.D. Leka ${ }^{2}$, Katharine K. Reeves ${ }^{1}$, K. E. Korreck ${ }^{1}$, L. \\ Golub $^{1}$, and E. E. DeLuca ${ }^{1}$
}

\begin{abstract}
We investigate the evolution of coronal loop emission in the context of the coronal magnetic field topology. New modeling techniques allow us to investigate the magnetic field structure and energy release in active regions. Using these models and high resolution multi-wavelength coronal observations from the Transition Region and Coronal Explorer (TRACE) and the X-ray Telescope (XRT) on Hinode, we are able to establish a relationship between the light curves of coronal loops and their associated magnetic topologies for NOAA Active Region 10963. We examine loops that show both transient and steady emission, and we find that loops that show many transient brightenings are located in domains associated with a high number of separators. This topology provides an environment for continual impulsive heating events through magnetic reconnection at the separators. A loop with relatively constant X-ray and EUV emission, on the other hand, is located in domains that are not associated with separators. This result implies that larger-scale magnetic field reconnections are not involved in heating plasma in these regions, and the heating in these loops must come from another mechanism, such as small-scale reconnections (i.e., nanoflares) or wave heating. Additionally, we find that loops that undergo repeated transient brightenings are associated with separators that have enhanced free energy. In contrast, we find one case of an isolated transient brightening that seems to be associated with separators with a smaller free energy.
\end{abstract}

Subject headings: Sun: corona — Sun: magnetic topology — Sun: UV radiation - Sun: X-rays

\footnotetext{
${ }^{1}$ Harvard-Smithsonian Center for Astrophysics, Cambridge, MA 02138

${ }^{2}$ NorthWest Research Associates, CoRA Division, Boulder, CO 80301
} 


\section{Introduction}

Observational studies of the character and evolution of coronal structures are becoming more refined as observational capabilities improve and accompanying models by which to interpret the results are developed. In studies of the evolution of coronal emission, observations at coronal-emission wavelengths show a variety of structures that evolve in distinct ways. It is useful to examine these observations in the context of the magnetic field. Coronal loops observed in soft X-ray and EUV have been studied since the Skylab observations in the 1970s (e.g., Vaiana et al. 1973; Yoshida et al. 1995; Berger et al. 1999). In previous studies, the spatial and temporal correlations between soft X-ray observations and EUV observations of coronal loops have been investigated in order to study coronal loop evolution (Schmieder et al. 2004; Nitta 2000; Nagata et al. 2003). Winebarger \& Warren (2005) found that structures observed in the X-rays and then subsequently in the EUV are due to emission from heated coronal loops cooling through the passband of each instrument.

Recent studies using the Hinode/EUV imaging spectrometer (EIS) have presented observations that show a brightening in EUV following a brightening in X-rays (Warren et al. 2007; Ugarte-Urra et al. 2009; Warren et al. 2009, 2010). A multi-thread hydrodynamic simulation has reproduced several coronal loop characteristics, including high electron density and a long observed life time, for the coronal loops near $1 \mathrm{MK}$, but it has difficulty reproducing the high temperature loop emission observed by XRT (Warren et al. 2009). It has also been shown that simple impulsive and quasi-steady heating does not reproduce the observed loop characteristics (Ugarte-Urra et al. 2006).

Several studies have shown evidence for magnetic field changes during the coronal loop evolution (Winebarger \& Warren 2005; Warren et al. 2007). Total magnetic flux in active regions has been used to investigate if the heating rate is proportional to a power of the total magnetic flux (Warren \& Winebarger 2006; Lundquist et al. 2008; Fludra \& Ireland 2008). Bulk active-region properties can provide statistical guidance (Fisher et al. 1998), but as Ugarte-Urra et al. (2009) show, different coronal emission lifetimes can be related to the field strength and its variation at loop boundaries. One recent study suggests that the changes in the topology of the magnetic field may be related with the evolution of the high temperature coronal loop in X-ray emission (Warren et al. 2009).

Starting from magnetogram observations, Magnetic Charge Topology (MCT) models (see Longcope 2005, for an overview) provide a framework to analyze the coronal topology, including the magnetic field connectivity and separator field lines. In this class of models, flux concentrations on the boundary are represented by point charges or sources. The magnetic field lines established by the MCT model form domains, defined as continuous volumes containing field lines that connect the same pairs of sources (Longcope \& Klapper 2002). 
Thus, the MCT model can determine which domains are along a given line of sight in a potential (current-free) extrapolation of the coronal magnetic field.

Domains are bounded by separatrix surfaces, whose intersections form separator field lines, and thus are the location of reconnection in MCT models. Priest et al. (2005) have proposed that slow photospheric motions can generate electric current near separators and separatrices which can be released as a heating energy. Recently, Noglik et al. (2009) showed that a potential magnetic field extrapolation represents the structure of the hotter X-ray loops and the larger cool loops seen in $171 \AA$ images appear to follow the separatrix surfaces determined by the MCT analysis. In addition, Plowman et al. (2009) show that separators explain the observed uniform width of coronal loops better than randomly distributed potential field lines. In this paper we use the MCT model to determine the location of flux domains and separators. By construction, this model disallows reconnection, but can identify the location of the separators where reconnection is most likely to occur. The details of how reconnection across the separators heats coronal loops in a flux system requires a more detailed time dependent MHD simulation that is beyond the scope of this paper.

The Minimum Current Corona (MCC) model (Longcope 1996) has been developed in order to determine the quasi-static evolution of magnetic fields starting with the potential fields of the MCT model and introducing currents along separators in response to footpoint motions. The MCC model represents the corona with the minimum permissible current after the evolution using a series of quasi-static steps and subject to the constraint that there is no reconnection (Longcope 1996). The resulting increase in energy above the potential field energy (free energy) can be released in the form of heating (Longcope 1996; Priest et al. 2000, 2005). This model has been applied to several flare events to investigate the energy source of the flares (Longcope et al. 2007; des Jardins et al. 2009).

In this paper, we use the MCC model to calculate the energy build-up from the photospheric magnetic field evolution, presenting this output in the context of multi-wavelength observations of the temporal evolution of the coronal emission. The MCT model is used to establish potential magnetic field connectivity, determine separators and domains along the line of sight at various coronal heights. The MCC model is used to determine coronal currents and quantify the energy buildup. The results of the MCC analysis are compared to the evolution of the coronal loop brightness, comparing selected areas of both steady emission and transient emission.

In $\S 2$, we describe the observational data employed in this analysis. In $\S 3$, we explain briefly the MCT and MCC models. We also describe the magnetic topology of AR 10963 as characterized by the MCT model and the free energy calculated by the MCC model in $\S 3$. In $\S 4$, we discuss the observed coronal loop brightness evolution as compared to the results 
of the MCT and MCC models. In $\S 5$, we present our conclusions.

\section{Observational Data}

The target of this study is NOAA Active Region 10963 (AR 10963), which transited the visible solar disk from 2007 July 8-23. This region is a very small, exceptionally quiet numbered active region, producing a few B- and small C-class flares (peak flux in the Geostationary Operational Environmental Satellite (GOES) 1-8 $\AA$ soft X-ray band of $\simeq 10^{-7}, 10^{-6} \mathrm{~W} \mathrm{~m}^{2}$, respectively). The region was event-quiet above the B-class threshold from July 11 00:05 UT until July 18 13:50 UT. The day of interest for this study was during the quiet time, focusing on 2007 July 14.

The X-ray telescope (XRT, Golub et al. 2007) on board the joint NASA/JAXA mission Hinode (Kosugi et al. 2007) observes the solar corona at high $\left(\sim 1^{\prime \prime}\right.$ pixels) spatial resolution in multiple bandpasses. For this study, we use a sequence of images from the $\mathrm{Al} /$ Poly filter, which samples plasma at $>2 \mathrm{MK}$, with a 20 s cadence from 18:27 UT on July 14 to 01:47 UT on July 15. The XRT observed AR 10963 with deep exposures in the C/poly (> 3 MK) filter to study faint structures until 18:15 UT on July 14. These earlier observations do not allow for study of the changes in coronal brightness since coronal loop images were saturated.

The Transition Region and Coronal Explorer (TRACE, Handy et al. 1999) observations are at a 1 minute cadence in the $171 \AA$ band, which samples the coronal plasma at $\sim 1 \mathrm{MK}$ with $\sim 0.5^{\prime \prime}$ pixels.

The line-of-sight component of the pixel-averaged photospheric magnetic field was obtained from the Michaelson Doppler Interferometer (MDI) aboard the Solar and Heliospheric Observatory (SoHO, Scherrer et al. 1995). For this study, a roughly 24 hour time sequence of the 96-minute cadence, $\sim 2^{\prime \prime}$ data was used, from 2007 July 14 01:39 UT to 2007 July 15 00:00 UT.

\subsection{Co-alignment among XRT, TRACE, and MDI}

Co-alignment between the datasets arising from three different instruments on three different spacecraft was crucial for this study, and was performed in the following manner.

We used the MDI level 1.8 data and full-sun, level 1 data from the Extreme-ultraviolet Imaging Telescope (EIT) on SoHO (calibrated by eit_prep.pro in the SolarSoft suite of analysis tools). We first supposed that an internal co-alignment between MDI and EIT is correct, 
and the EIT images then provided the common coalignment platform for both the XRT and TRACE data. Active region 10963 was observed by XRT and TRACE for $\sim 6$ hours with $\mathrm{Al} /$ Poly (1.0286" pixels) and $171 \AA$ (0.5" pixels), respectively. The EIT (2.63" pixels) observed the full sun every 6 hours at $284 \AA$ and $171 \AA$.

The XRT observed the full solar disk with Ti/poly every 20 minutes as a context image. X-ray bright points on the full Sun at 19:07:15 UT on July 14 were aligned with EIT $284 \AA$ at 19:06:07 UT on July 14. Due to the similar response to high temperature plasma, the EIT $284 \AA$ and the XRT Ti/poly observations were used to co-align the satellites. The spacecraft jitter on Hinode (Shimizu et al. 2007) was corrected with the reference image at 19:07:15 UT.

The TRACE $171 \AA$ observation at July 14 18:59:26 UT was aligned with the EIT $171 \AA$ observation at July 14 19:00:13 UT. The aligned image was used as a reference to align for later time observations for $\sim 6$ hours (using trace_cube_pointing.pro in SolarSoft) to account for jumps in the TRACE pointing as the active region was tracked across the disk.

\subsection{Area selection for light curves}

We select four areas in the XRT and TRACE data with which to investigate coronal brightness changes. These areas are presented as four boxes in Figure 1. Inner and outer boxes correspond to approximately $5^{\prime \prime} \times 5^{\prime \prime}$ and $10^{\prime \prime} \times 10^{\prime \prime}$ areas, respectively. The Boxes 1 , 2 , and 4 were selected based on the coronal loops in the XRT observation, and Box 3 was selected based on a small loop visible in the TRACE data at 22:24 UT on July 14 .

Light curves for each box are presented in Figure 2, Box 1 shows a brightening in the TRACE observation about an hour and a half after a decrease in X-ray brightness. Box 2 shows transient brightenings in XRT, however the TRACE light curve shows only very small changes in brightness. Box 3 shows transient brightenings in both XRT and TRACE. As seen in the animation version of Figure 1, the light curves of Boxes 2 and 3 include the brightness changes for several loops. Box 4 shows steady emission in both XRT and TRACE, at a lower level in XRT than any of the other boxes. The light curves in Figure 2 show similar behaviors for the two box sizes, which verifies that the light curves do not depend on a small change in the location of the box.

Figure 1 also shows a loop (L1) that contributes to the light curves in Box 1 on the XRT and the TRACE observations at 22:24 UT and 23:48 UT, respectively, when the brightenings are seen in each instrument. The ends of the loops seen by the XRT observation are represented with F1. These locations are used to estimate the height of the loop in $\S 4.1$ (see also Table 4 in Appendix). The location of the loop for the Box 1 is selected based on the 
loop observed by the TRACE because the loop is better resolved in the EUV observation, and then the same location is represented on the XRT observation.

\section{Magnetic Topology and Energy Buildup}

We utilize a Magnetic Charge Topology model, and the Minimum Current Corona model to understand the magnetic topology and the magnetic energy changes in the active region that we observe with XRT and TRACE. We use the MCT model to define the magnetic topology and locate possible sites for magnetic energy reconnection. With the MCC model, we quantify the magnetic energy changes. The details of these models and our implementation of them for the analysis of AR 10963 are described in the next subsections.

\subsection{Magnetic Charge Topology model}

The MCT model is used to establish the magnetic connectivity by potential magnetic field extrapolation into the corona from a distribution of point sources representing the photospheric magnetic field distribution (Baum \& Bratenahl 1980; Gorbachev \& Somov 1988; Priest \& Forbes 1989; Lau 1993; Démoulin et al. 1994; Parnell et al. 1994). As a result of the extrapolation, the magnetic field lines form a domain, defined as a volume containing field lines that have the same sources at their ends (unless the field lines tend to infinity). In the following paragraphs, we explain several topological terms derived from the inferred magnetic connectivity that we use in this paper.

Locations where the magnetic field vanishes are referred to as magnetic null points. In three dimensional geometry, the local magnetic structure of a null can be described in terms of a fan surface and two spine field lines (Parnell et al. 1996). The fan surface is a set of field lines which radiate out from a null point (positive, B-type null) or radiate into a null point (negative, A-type null). The spine field lines are those directed toward the positive null point or directed away from the negative null point.

The fan forms a separatrix surface that divides the corona into volumes of different connectivity called domains. Separators are field lines which begin at a positive null and end at a negative null, and they are located at the intersection of separatrix surfaces. Magnetic reconnection and energy release in a coronal field stressed by photospheric motions are likely to occur near separators, although it should be noted that the MCT model does not allow for reconnection.

The flux in a connection $\left(\psi_{i j}\right)$ is determined from the number of magnetic field lines 
initiated from the source $(i)$ in random directions and followed to their termination at a source of the opposite polarity $(j)$ (Barnes et al. 2005). For each pair of sources, it is possible to have connections through multiple domains (Longcope \& Klapper 2002). In this analysis, each different pair of sources defines a single domain. Thus the connectivity flux and the domain flux are the same for each pair of sources in this work.

\subsection{Partitioning the magnetogram time-series}

To extrapolate the magnetic field lines into the corona, the MCT model requires a set of distinct point sources derived from an observed magnetic field. Therefore, the observed magnetic fields must be partitioned in a way that represents the surface magnetic flux concentrations. To determine the flux concentrations from the photospheric magnetic field observations, the observed magnetic features are partitioned to preserve important magnetic morphology, and the location of each source is determined by the flux weighted center of the partition (Barnes et al. 2005).

We use a time series of 96-minute-cadence MDI magnetograms for the photospheric magnetic field observation. First, we select the region of AR 10963 from the full-disk magnetograms for $\sim 24$ hours, taking into account solar rotation. The selected area retains the balance of positive and negative sources within a $4 \%$ ratio of the total signed to total unsigned magnetic line of sight signal. The selected regions are transformed from the image plane (or "plane of the sky") to a plane that is tangent to the Sun's surface at the flux weighted center of the selected active region. The " $\mu$-correction" is applied, which assumes that all field is radial, and divides the line-of-sight magnetogram by the cosine of the observing angle, to approximate the radial field. This process transforms the observed line-of-sight pixel-averaged magnetic field component to a tangent plane approximation of the magnetic flux, as required by the MCT model.

The top panel of Figure 3 shows the boundaries which result from partitioning the positive and negative sources. We initially determine the flux concentration at the last time observed, July 15 00:00 UT, since an earlier study has found that performing the partitioning in reverse chronological order provides the most consistent partitioning in light of noise and solar evolution (Longcope et al. 2007). The partitioning parameters used are similar to those in Barnes et al. 2005, though they have been adjusted to match this specific active region!

\footnotetext{
${ }^{1}$ Specifically, for this dataset we employ a field threshold of 3 times the detection threshold, a smoothing depth of $0.5 \mathrm{Mm}$, a saddle point merging level of $200 \mathrm{G}$, and a minimum of source flux of $10^{4} \mathrm{G} \mathrm{Mm}^{2}$ (see Barnes et al. 2005, for a description of these parameters).
} 
Recently, Longcope et al. (2009) found that there are only slight differences in connectivity that result from using different partitioning parameters, so our results are unlikely to be sensitive to the choice of partitioning parameters. The bottom panel of Figure 3 shows domain fluxes among all sources at the same time as the top panel.

MDI 96-minute data consist of images with integration times of 30 and 300 seconds during the 24 hour period of interest, and different integration times could conceivably change the inferred flux. To mitigate this effect, the detection level was assigned to be the $3 \sigma$ level of a Gaussian fit for the histogram of each MDI observation. This procedure sets an appropriate background level for each integration time. No significant systematic difference was detected in the resulting fluxes above the $3 \sigma$ threshold.

Beginning from the end of the time series, the observed magnetic elements in the magnetograms are advected to the earlier MDI observations using Fourier Local Correlation Tracking (FLCT, Fisher \& Welsch 2008), in reverse chronological order for the same reasons as mentioned above. The FLCT finds the velocities of features in two successive images by computing the cross-correlation function using standard Fast Fourier Transform techniques (Welsch et al. 2004)2. These velocities are horizontal velocities for each pixel in the MDI observations. The computed velocities are then used to track elements and determine their partitioning assignments in subsequent observations. Thus, this procedure preserves the partitioning information as consistently as possible through the time series.

In Figure 4, we show a plot of the total unsigned flux for all sources obtained by the timeseries partitioning, and the time-averaged total flux. It is clear that there is little variation during the $\sim 24$ hours, implying that there is no significant emerging or submerging flux during this time period. Therefore, we impose that the flux for each source is also constant in time, since the flux changes that do occur are too small to contribute significantly to the active region field. We use the time-averaged flux for each source in the MCT model. This approach prevents the transfer of flux between neighboring sources in the partitioning, and precludes changes in connectivity due to changes in source flux resulting from emergence or submergence, thus simplifying the usage of the MCC model (Longcope et al. 2007), as described in $\S 3.4$.

The source N4 (near [220", -100"] in Figure 3) is submerged at 17:36 UT on July 14, and only appears in 4 time steps. The average flux of N4 for the four sequential MDI observations when it is above threshold is $2.6 \times 10^{20} \mathrm{Mx}$. However the average fluxes of other sources are in the range of $(4.9-67) \times 10^{20} \mathrm{Mx}$. Therefore, we do not consider the source N4 for this

\footnotetext{
${ }^{2}$ For the implementation of the FLCT here, we employ time differences between two images of 1 , unit of length of a single pixel of 1, and sub-images weighted by Gaussian width of 45, see Fisher \& Welsch (2008).
} 
analysis on account of its relatively small flux and short lifetime. This choice helps the analysis stay consistent with the assumption that there is no emerging/submerging flux.

In Figure 5 we demonstrate the evolution of partitioning, source locations, and selected connection fluxes $\left(\psi_{i j}\right)$ calculated by the MCT model as described in $\S 3.1$, for the first and last time. Only connections related to the seven separators discussed at length below are shown. In this figure, the change in the source locations between the first time and the last time can be compared. For instance, it can be seen that P3 and N6 are approaching with a rotation during $\sim 24$ hours indicating that this region is generating significant currents (see details in $\S 3.4)$.

\subsection{Magnetic topology of AR 10963}

The MCT model was used to establish the magnetic topology of AR 10963 at 00:00 UT on July 15. The flux concentrations obtained from the MDI observations by the partitioning explained in the previous section were used as a set of distinct sources. Figure 6 shows seven of the separators for this active region, specifically those which are topologically related to the areas in the light curves shown in Figure 2.

In order to track the build up of currents along the separators, it is useful to represent the domain fluxes $\left(\psi_{i j}\right)$ in terms of the source fluxes $\left(\Phi_{k}\right)$ and the separator fluxes $\left(\Psi_{\sigma}\right)$, where the indices $i, j$ and $k$ refer to sources while the index $\sigma$ refers to separators. Because all the sources are in the plane of the photosphere, there is a "mirror corona" below the photosphere, which is a reflection of the corona. Thus for each separator, there is a mirror separator, and the two form a closed curve. To express the domain fluxes in terms of the source and separator fluxes, it is convenient to first determine which domains pass through the closed curves formed by each separator and its mirror.

To illustrate how to determine which domains pass through such a closed curve, consider separator 5 (hereafter $\Psi_{5}$ ) which starts and ends at the nulls B11 and A3, respectively, and consists of the intersection of the separatrix surfaces from these two nulls. The separatrix surface associated with null A3 intersects the photosphere along the fan trace from A3 to source P4, then along the spines of null B8 to source P2, then along the spines of null B11 to source P3, and returns to null A3 along the fan trace from P3. This surface encloses only source N6, thus all field lines within this separatrix surface must terminate on N6. Similarly, the separatrix surface associated with null B11 only encloses source P3, thus all field lines

within this separatrix surface must start on source P3. Since $\Psi_{5}$ is the intersection of these separatrix surfaces, the domain P3-N6 passes through the closed curve consisting of $\Psi_{5}$ and 
its mirror image below the photosphere.

Alternatively, one can consider the photospheric footprint of domain P3-N6. Since all the sources lie in the plane of the photosphere, any field line originated in this plane must, by symmetry, remain in this plane. One can see in Figure 6 that any field line initiated at source P3 in the photosphere must pass under $\Psi_{5}$ in order to terminate on source N6, and hence the domain P3-N6 passes through the closed curve of $\Psi_{5}$ and its mirror.

As a somewhat more complicated example, consider $\Psi_{3}$, whose endpoints are on nulls B6 and A2. The separatrix surface associated with B6 encloses only source P5, but the separatrix surface associated with A2 encloses both source N3 and source N5 (Note that source N5 is also enclosed by the separatrix surface associated with null A1, and this separatrix surface is, in turn, enclosed by the separatrix surface from null A2, but the important point is that source N5 is enclosed by the separatrix surface from A2). Thus, domains P5-N5 and P5-N3 both pass through the closed curve consisting of $\Psi_{3}$ and its mirror. Once again, one can instead consider the photospheric footprint of these domains. Any field lines initiated in the photosphere from P5 must pass under $\Psi_{3}$ to reach source N5, and similarly for field lines which terminate on source N3. The domains enclosed by the separators and the nulls where the separators start and end are shown in Table 1.

In addition to the domains associated with each separator, each null whose fan is unbroken (that is, each null for which all field lines in the fan surface end on the same source and thus is not the start/end of a separator; see Longcope \& Klapper (2002)) implies the existence of a domain with flux equal to the flux of the spine source enclosed by this domain. For example, null B10 has an unbroken fan, and so the flux in domain P6-N1 equals the flux of source P6. This constrains those domain fluxes not associated with any separator.

Knowledge of the domains passing through the closed curves of the separators and their mirrors, combined with knowledge of the nulls with unbroken fans is sufficient to express the domain fluxes in terms of the separator and source fluxes. The flux in each domain not enclosed by any separator is determined from conservation of the source fluxes, $\Phi_{i}=\sum_{j=1}^{n} \psi_{i j}$, where $\Phi_{i}$ is the source flux determined from the observed magnetic field obtained by the partitioning process, $\psi_{i j}$ is the domain flux between the $i^{\text {th }}$ and $j^{\text {th }}$ sources, and $n$ is the total number of sources of the opposite polarity to source $i$. The result, shown in Table 2, provides information about which domains can be affected by each separator. This information on the relationship between domain fluxes and separator fluxes is used to interpret our results in $\oint 4$. 


\subsection{Minimum Current Corona model}

In the previous section, we established the magnetic topology of AR 10963 using the MCT model which assumes that the coronal magnetic field is a potential (current-free) field. Longcope (1996) developed the MCC model to incorporate source evolution, current generation along separators, and energy build-up in the context of the MCT model. We utilize the MCC model to quantify the magnetic free energy of AR 10963 in $\$ 3.5$. In this section, we describe briefly the MCC model (Longcope 1996, 2001).

As a constraint in the MCC model, Longcope (2001) has proposed a flux-constrained equilibrium (FCE). This equilibrium is found by minimizing the magnetic energy subject to the constraint that, in the absence of emergence and submergence of flux through the boundary, the domain matrix does not change. This constraint is equivalent to prohibiting reconnection in the corona. The minimum energy magnetic field occurs when current flows only along separator field lines, such that each domain is current-free (potential) but is encircled by current ribbons that cancel the non-potential flux introduced by footpoint motions.

In a three dimensional magnetic field, the currents occur at points topologically equivalent to separators in the potential field (Longcope \& Cowley 1996; Longcope \& Klapper 2002). Therefore, the current in the minimum energy state can be found using the flux through a separator. In the minimum energy state, the flux $\left(\Psi_{\sigma}\right)$ through a separator $(\sigma)$ can be expressed as (Longcope 1996)

$$
\Psi_{\sigma}=\Psi_{\sigma}^{(v)}+\Psi_{\sigma}^{\left(I_{\sigma}\right)}+\sum_{\sigma^{\prime} \neq \sigma} \Psi_{\sigma}^{\left(I_{\sigma^{\prime}}\right)}
$$

where $I_{\sigma}$ is the current flowing along a separator $\sigma$. The vacuum term $\left(\Psi_{\sigma}^{(v)}\right)$ is the sum of the domain fluxes enclosed by the separator in the potential field (see §3.3). The second term on the right hand side, $\left(\Psi_{\sigma}^{\left(I_{\sigma}\right)}\right)$ is a self-flux from the current along the separator. The third term is the flux from other separator currents.

At the first time $\left(t_{0}\right)$, we assume that there is no current. Then, from Equation 1 we get

$$
\Psi_{\sigma}\left(t_{0}\right)=\Psi_{\sigma}^{(v)}\left(t_{0}\right)
$$

Using the constraint on the domain flux that there is no connectivity change, Equation 1 becomes, at $t_{0}$ and later time $t_{1}$,

$$
\Psi_{\sigma}\left(t_{0}\right)=\Psi_{\sigma}^{(v)}\left(t_{0}\right)=\Psi_{\sigma}\left(t_{1}\right)=\Psi_{\sigma}^{(v)}\left(t_{1}\right)+\Psi_{\sigma}^{\left(I_{\sigma}\right)}\left(t_{1}\right)+\sum_{\sigma^{\prime} \neq \sigma} \Psi_{\sigma}^{\left(I_{\sigma^{\prime}}\right)}\left(t_{1}\right) .
$$


Therefore, the flux difference in the potential field $\left(\Delta \Psi_{\sigma}^{(v)}=\Psi_{\sigma}^{(v)}\left(t_{1}\right)-\Psi_{\sigma}^{(v)}\left(t_{0}\right)\right)$ should be canceled by the sum of the second and the third terms in the right side of Equation 3 because the $\Delta \Psi_{\sigma}$ is zero with no connectivity changes.

In order to determine the current along the separator field line, the MCC model requires a relationship between the self-flux and current. The self-flux produced by current along the separator (Longcope 1996; Longcope \& Magara 2004) is given by,

$$
\Psi_{\sigma}^{\left(I_{\sigma}\right)}=\frac{I_{\sigma} L_{\sigma}}{c} \ln \left(\frac{e I_{\sigma}^{*}}{\left|I_{\sigma}\right|}\right)
$$

where $e$ is the base of the natural logarithm, $I_{\sigma}$ is the current along separator, $L_{\sigma}$ is the length of the separator in potential field, and $I_{\sigma}^{*}$ is a characteristic current proportional to the average perpendicular magnetic shear along separator (see Longcope \& Magara 2004 for details).

The third term on the right hand side in Equation 1, the flux from other separator currents, is expressed with a mutual inductance matrix $\left(M_{\sigma \sigma^{\prime}}\right.$, Longcope 1996; Longcope \& Beveridge 2007),

$$
\sum_{\sigma^{\prime} \neq \sigma} \Psi_{\sigma}^{\left(I_{\sigma^{\prime}}\right)}=\sum_{\sigma^{\prime} \neq \sigma} M_{\sigma \sigma^{\prime}} \frac{I_{\sigma^{\prime}}}{c}
$$

assuming that two separators are far enough apart, so the change in the flux enclosed by separator $\sigma^{\prime}$ will not be greatly affected by the internal distribution of the current on $\sigma$. Therefore, the current along the separator field line is calculated by

$$
\Delta \Psi_{\sigma}^{(v)}=\frac{I_{\sigma} L_{\sigma}}{c} \ln \left(\frac{e I_{\sigma}^{*}}{\left|I_{\sigma}\right|}\right)+\sum_{\sigma^{\prime} \neq \sigma} M_{\sigma \sigma^{\prime}} \frac{I_{\sigma^{\prime}}}{c} .
$$

The above equation is used to find the current $I_{\sigma}$, and thus the energy change for each separator, $\Delta W_{M C C}$, can then be calculated from the following

$$
\Delta W_{M C C}=\frac{1}{4 \pi} \int_{\Psi_{\sigma}^{(v)}}^{\Psi_{\sigma}} I_{\sigma} d \Psi_{\sigma}=\frac{L_{\sigma} I_{\sigma}^{2}}{2 c^{2}} \ln \left(\frac{\sqrt{e} I_{\sigma}^{*}}{\left|I_{\sigma}\right|}\right)+\frac{I_{\sigma}}{2 c^{2}} \sum_{\sigma^{\prime} \neq \sigma} M_{\sigma \sigma^{\prime}} I_{\sigma^{\prime}}
$$

From the above equations, we evaluate the current along each separator field line which arises due to footpoint motions, and the inferred excess energy over the potential field, referred to as the free energy. This free energy is available to be released in the form of localized heating (e.g., Longcope 2001; Priest et al. 2000, 2005). 


\subsection{Magnetic energy of AR 10963}

We utilize the MCC model to investigate the magnetic energy changes of AR 10963. The evaluated magnetic energy changes are compared with the coronal loop brightness evolution observed by XRT and TRACE in $\S 4$.

The MCC model infers the current and the free energy by using the changes of the potential field flux through the separators as described in the previous section. However, the magnetic topology evolves with time, so separators that are present at one time are not necessarily present at the next (e.g., des Jardins et al. 2009). Therefore one cannot follow the same separator for the entire time series, and a different approach is needed to determine the change in flux enclosed by each separator.

The fundamental assumption of the MCC model is that there is no magnetic reconnection in the corona, thus the flux in each domain must remain constant. As described in $\S 3.3$ and shown in Table 1, we have related the flux enclosed by each separator to the flux of the set of domains enclosed by the separator. Therefore, the flux through separators used in the MCC model has been replaced by the appropriate combination of domain fluxes, smoothed with a boxcar function with a width of 3 time steps at each time to reduce the noise due to partitioning.

We evaluate the magnetic energy changes assuming that the magnetic field at the first time, July 14 01:39 UT, is a potential field with no current. Then, the domain fluxes are used to determine the changes in separator flux used in Equation 6 to derive the currents, and Equation 7 is used to determine the free energy. In the initial stages, the model does not represent the coronal currents well, since the system is started in a potential state, but it should improve as it evolves away from the potential state. Since the energy evolves slowly over several hours in this active region, the choice of different initial time within a few hours will not affect the results of our energy calculation.

In Figure 7 we show the free energy resulting from current along the seven separators shown in the topology map in Figure 6. Separators $\Psi_{1}$ and $\Psi_{2}$ show a steady increase in the free energy after $\sim 15: 00$ UT. Separators $\Psi_{3}$ and $\Psi_{7}$ show no significant changes in energy from the initial potential state; thus those curves stay near the x-axis in Figure 7 , As seen in Figure $6, \Psi_{3}$ and $\Psi_{7}$ are nearly co-located and enclose many of the same domains, so their free energies behave similarly. Separator $\Psi_{4}$ shows an increase in energy until $\sim 13: 00 \mathrm{UT}$, and then a decrease until the last time. Separator $\Psi_{5}$ shows an increase in energy from 18:00 UT and then a decrease. Separator $\Psi_{6}$ shows a larger increase from $\sim 18: 00$ UT.

The mutual inductance term used in this analysis does not account for the internal distribution of current along each separator (see §3.3). Separators $\Psi_{3}$ and $\Psi_{7}$ are located 
close together as seen in Figure 6, but the free energies are very small. Therefore, we assume that the mutual inductance is not important for the overall energetics of the system.

\section{Magnetic energy buildup and coronal loop brightness evolution}

We have evaluated the free energy build-up along separators in AR 10963 over a 24 hour time period using the MCC model derived from a series of 15 times of MDI observations. This free energy can be released through localized heating and rapid magnetic reconnection (Longcope 2001). We have also introduced the brightness evolution of coronal loops observed by XRT and TRACE. The free energy of AR 10963 is now compared to the observations in the Soft X-rays and EUV to determine if the free energy is related to the coronal loop brightening.

In Figure 2, we show the light curves for four locations selected from the coronal loop observations of Hinode/XRT and TRACE. Box 1 shows a brightening in XRT shortly followed by a brightening in TRACE. Boxes 2,3 show many transient brightenings in both telescopes. Box 4 shows steady emission in both XRT and TRACE. We compare these light curves to the free energy buildup for $\sim 24$ hours as obtained by the MCC model.

We compare the locations of separators with the coronal loops of AR 10963 in Figure 8 , where the magnetic topology of AR 10963 is superimposed on the XRT and TRACE images at 23:54 UT on July 14 viewed on the image plane, and the separators and light-curve box locations are also shown. In this section, we first investigate which domains lie along the line of sight at various coronal heights for each of the four light curve boxes. Second, we discuss the coronal loop brightness evolution in comparison with the domains and separators.

\subsection{Domains along the line of sight}

The optically thin X-ray and EUV emission seen by XRT and TRACE is due to contributions from plasma along the line of sight of the observations. The MCT model is useful for determining which domains lie along the line of sight through the corona, at various heights above the photosphere. We determine which domains intersect the line of sight at the locations of the four light curve boxes in order to map which domains contribute to the observed emission.

The left panel of Figure 9 shows the footprint of domains on the photosphere, along with the four light curve boxes $\left(10^{\prime \prime} \times 10^{\prime \prime}\right)$. Each footprint is bounded by spines and fan traces, shown as black thin and dashed lines, respectively. Each color represents a different 
domain which is shown on the right side of the Figure with the same color. The right panel of Figure 9 shows potential field lines with the same colors on the left panel. The potential field lines are shown on the XRT image as in Figure 8 .

Figure 10] shows the domains as a function of height along the line of sight from each of the four boxes where the light curves were calculated. Each box has four points on the horizontal axis that represent the four corners (SE, NE, SW, NW in that order) of the $10^{\prime \prime} \times 10^{\prime \prime}$ boxes used to calculate the light curves. The flux domains are evaluated every $0.1 \mathrm{Mm}$ from the photosphere.

Some domains in the left panel of Figure 10 are not readily evident in the left panel of Figure 9. The P2-N2 domain is not seen in the left panel of Figure 9 because the domain does not have a photospheric footprint, i.e., it is a coronal domain (Beveridge \& Longcope 2005). The P5-N2 domain is located between domains P5-N3 and P5-N1. It is very thin and can be seen only near $\left(280^{\prime \prime},-150^{\prime \prime}\right)$ in Figure 9, and near $50 \mathrm{Mm}$ of Box 1 in the bottom panel in Figure 10.

We show the domains for each light curve box in the second column in Table 3 . The domains are listed in order of coronal height from the photosphere. For instance, the light curve in Box 1 receives contributions from P5-N3, P5-N1 and P5-N2 below P2-N1, although P5-N2 occupies only a very thin layer near $50 \mathrm{Mm}$.

To determine which domains make the main contribution to each light curve, we need to know the height range of the loops contributing to the light curves. We estimate the height of a loop by assuming that the loop is a semicircle lying in a plane perpendicular to the solar surface, with diameter equal to the distance between the two ends of the loop seen by XRT and TRACE (see $\S 2.2$ and Appendix). Using this method, we find that the loop that clearly contributes to the light curve in Box 1 has a height of $\sim 50 \mathrm{Mm}$. This number takes into account the projection due to the location of the AR $10963 \sim 250^{\prime \prime}$ solar west of central meridian. We estimate the heights of loops that contribute to the light curves in Box 2 - 4 (see Figure 11 and Appendix) and find that the estimated heights are all lower than $50 \mathrm{Mm}$ (see Table 4). Therefore we assume that the heights of coronal loops that produce the enhancements in the light curves are lower than $\sim 50 \mathrm{Mm}$. Loops in the core of active regions tend to be relatively short, so this is a reasonable assumption. Using this assumption, we only include domains that are lower than $\sim 50 \mathrm{Mm}$ in the analysis.

In the high corona above $\sim 50 \mathrm{Mm}$, the domains P4-N1, P7-N1, and P1-N1 occupy the corona for all boxes. In addition, the domain P2-N1 extends over Boxes 1, 2, \&3, but not Box 4. Loops contained in these high domains would mostly be very long, as can be seen in the potential field extrapolation in the right panel of Figure 9. Assuming these loops follow 
RTV scaling laws (Rosner et al. 1978), the density in them would be quite low because of their long length. Thus, we assume that the domains in the corona above the estimated height of the observed loops do not significantly affect the enhancements in light curves. The domains below $\sim 50 \mathrm{Mm}$ are represented with bold letters for each light curve box in Table 3.

\subsection{Relationships among the light curves, domains, and separators}

In this section, we investigate which separators can affect those domains that intersect the lines of sight for the light curves of the XRT and TRACE emission. This result is key to investigating if the free energy calculated by the MCC model can explain the coronal loop brightness evolution.

Table 2 shows the separators that can affect each domain (see $\S 3.3$ ). We show the relevant separators next to each domain in Figure 10, Table 3 summarizes the relationships between the light curve boxes, domains, and separators. The separators that can affect the domain flux are shown in the third column in Table 3. As we described in the previous section, we consider the domains below $\sim 50 \mathrm{Mm}$.

The light curves of Box 1 show a transient brightening in the TRACE observation after a decrease of intensity in the XRT observation, implying that there is a cooling of the coronal loop from X-ray temperatures $(\gtrsim 2 \mathrm{MK})$ through EUV temperatures $(\sim 1 \mathrm{MK}$, see Figure 2). Observations of a coronal loop brightening in EUV following a loop brightening in $\mathrm{X}$-rays have been examined previously. Many authors have studied the correlation between X-ray and EUV brightenings and the implications of this correlation for the loop heating mechanism (Winebarger \& Warren 2005; Ugarte-Urra et al. 2009, 2006; Warren et al. 2009). Winebarger \& Warren (2005) study several loops that are relatively seclude and uniquely shaped so that they are able to follow the same loop as it cools through the different filters. They conclude that the EUV emission can be produced by a loop that emits in the X-rays several hours before, although the delay time is difficult to reproduce through hydrodynamic modeling.

We have evaluated an approximate cooling time of the loop including the light curve Box 1 in Figure 1. Assuming that plasma is at a temperature of $10 \mathrm{MK}$ when it peaks in $\mathrm{XRT}$, and is at $1 \mathrm{MK}$ when it peaks in TRACE, and the loop is semicircular with a radius of $50^{\prime \prime}$, width of $10^{\prime \prime}$ and has a uniform cross section. With these assumptions, the density at the time of peak intensity in TRACE is about $2 \times 10^{9} \mathrm{~cm}^{-3}$, and the loop has a cooling time on the order of about an hour to go from $10 \mathrm{MK}$ to $1 \mathrm{MK}$. The temperature and 
density evolution are calculated using an analytic solution to the 1D hydrodynamic energy equation (Cargill et al. 1995), and the assumptions are not to be believed in detail. However this calculation is a reasonable zeroth order estimate for the cooling time. Comparing the estimated cooling time with the light curve of Box 1 (Figure 2) that shows about 2 hours between peak in XRT and TRACE, it is likely that the loop is cooling from XRT to TRACE temperatures. Thus we conclude that the emission in Box 1 is probably due to a cooling loop.

The light curves in Box 1 are integrated over domains P5-N3, P5-N2, and P5-N1 below $\sim 50 \mathrm{Mm}$. These domains are related to the separators $\Psi_{1}, \Psi_{3}$, and $\Psi_{7}$. The latter two separators, $\Psi_{3}$ and $\Psi_{7}$, have relatively little free energy with very little change as shown in Figure 7. Although the domain P5-N3 associated with $\Psi_{1}$ is along the line of sight of Box 1, the geometry of this separator indicates that it is probably not a key component of the emission in this area. In the right panel of Figure 9, the loop that is included in Box 1 is located in a region dominated by the magnetic field lines of domains P5-N3 and P5-N1. In addition, the height of $\Psi_{3}$ and $\Psi_{7}$ are both $50 \mathrm{Mm}$, and their lengths are both about $160 \mathrm{Mm}$. In contrast, $\Psi_{1}$ is much lower and shorter, with a height of $27 \mathrm{Mm}$ and a length of $89 \mathrm{Mm}$. A few studies have found that longer coronal loops lead to longer delay times between the appearance of the X-ray loop and the appearance of the EUV loop (Ugarte-Urra et al. 2006; Aschwanden et al. 2003). The delay time between the XRT and TRACE emission peaks in Box 1 is relatively long, thus the emission is likely to have come from longer loops that are located higher in the corona. The separators $\Psi_{3}$ and $\Psi_{7}$ are more likely associated with long loops than $\Psi_{1}$, so the emission in Box 1 is probably related to separators $\Psi_{3}$ and $\Psi_{7}$.

Therefore, our analysis shows that the emission variation in the area of Box 1 as summarized by its light curves most likely comes from the domains associated with the separators $\Psi_{3}$ and $\Psi_{7}$, which have a small free energy. Thus it is possible that the loop is in a nearly potential environment, so that not much energy release can occur. This small free energy may be the reason why there are not many repeated transient events in Box 1, as there are in Boxes 2 and 3.

Boxes 2 and 3 are related to domains P2-N3, P2-N2, P3-N3, and P2-N1. These domains are related to separators $\Psi_{1}, \Psi_{2}, \Psi_{3}, \Psi_{4}, \Psi_{6}$, and $\Psi_{7}$. Boxes 2 and 3 display several transient brightenings in Figure 2, Thus it is likely that the variable emission in these areas comes from the domains associated with a high number of separators, including, but not limited to, the separators for Box 1. Additionally the free energy associated with $\Psi_{1}, \Psi_{2}$, and $\Psi_{6}$ increases over the time period of the observations, indicating that the fields associated with these separators are stressed and non-potential. This configuration could provide an environment for continuous impulsive events in Boxes 2 and 3 through multiple reconnection 
processes occurring at the separators.

Recent studies have shown that continuous impulsive heating events are common in loops located in the core of an active region (Ugarte-Urra et al. 2009; Warren et al. 2007). Warren et al. (2007) presented bi-directional flows as evidence of magnetic reconnection during the evolution of coronal loops in the XRT and the EIS observations. The loops observed by Winebarger \& Warren (2005) shift positions as they cool, which may be evidence for a previous reconnection event. Therefore, our conclusion that areas of active regions that show impulsive emission are probably related to reconnection events along separators is broadly consistent with previous observations.

Transient brightenings in this active region during the observation period have different characteristics. The light curves of Box 3 show transient brightening in both XRT and TRACE. However, the light curves for Box 2 show transient brightenings in the XRT, but the TRACE light curve shows only very small changes. This situation may indicate that loops in Box 2 are being heated more frequently and have insufficient time to cool into the TRACE passband before being reheated. This kind of difference can not be addressed by our analysis since we only locate the possible sites for reconnection, and do not determine the frequency with which reconnection takes place.

The light curves of Box 4, which shows steady emission in both XRT and TRACE, intersect domain $\mathrm{P} 6-\mathrm{N} 1$ below $\sim 50 \mathrm{Mm}$. This domain does not have any associated separators. "Steady heating" has been suggested as the heating mechanism for loops that have TRACE moss at their footpoints, as the loop in Box 4 does (Antiochos et al. 2003; Warren et al. 2008). In this analysis, the emission of Box 4 comes from a domain that is not associated with any separators, indicating that larger-scale reconnection events are unlikely to be contributing to the heating of this plasma. Thus the steady heating proposed by Antiochos et al. (2003) is not likely to be due to reconnection events at separators shown in the larger-scale magnetic field configuration. Rather, the source of the heating that results in the emission observed in Box 4 may be due to many small reconnection events or nanoflares from local field tangling (e.g. Antiochos et al. 2003; Parker 1983b,a, 1988; Klimchuk 2006), or alternatively, MHD waves (e.g. Kumar et al. 2006; Ofman \& Wang 2008; Antolin \& Shibata 2010).

Several studies for the heating mechanism of coronal loops have difficulty explaining the brightness of observations in both EUV and X-ray emission and the delay between the appearance of X-ray and EUV loops (Warren \& Winebarger 2006; Ugarte-Urra et al. 2006). However, recent studies have pointed out the importance of magnetic properties to explain the coronal loop evolutions (Ugarte-Urra et al. 2009; Warren et al. 2009). Our result also concludes that the magnetic topology plays a significant role for the study of the heating mechanism of non-flaring coronal loops. 


\section{Conclusion}

In this analysis, we examined Hinode/XRT and TRACE observations of particularly well-resolved coronal loops in an active region that show different characteristics in their emission properties. In order to understand these differences, we compare the coronal loop evolution represented by four light curves from XRT and TRACE observations to the free energy calculated by the Minimum Current Corona (MCC) model. We compare the free energy gained from the motions of the magnetic footpoints with emission patterns that include steady emission and transient emission. This analysis marks the first time that the MCC model has been applied and tested as a full time series analysis for a quiet active region to investigate coronal loop evolution in plasma of different temperatures.

This work shows that the magnetic topology of an active region can provide crucial information for understanding the evolution of coronal loop brightness. One of the major questions in coronal loop evolution studies is the difference in the heating mechanism for steady emission versus transient emission. We find that regions which have very dynamic emission are related to a high number of separators that have enhanced free energy, indicating that reconnections along these separators may play a role in repeated transient heating events. In contrast, the light curve in Box 1 that shows a single transient brightening in the XRT observations followed by a brightening in TRACE is related to separators with little free energy. This relationship indicates that the fields in the vicinity of Box 1 are nearly potential to begin with. Thus there is not a lot of excess energy available for release through repeated reconnection events in this region, and repeated transient events are not observed in the light curve.

Finally, the steady emission in both XRT and TRACE comes from the domains that are not associated with any separator, indicating that reconnections involving the larger-scale field configuration are probably not associated with steady heating, and implicating either nanoflares or MHD waves as a possible heating source for these loops. We have found a topological explanation for the two classes of coronal loops, those that emit steadily and those that show transient emission properties.

The Solar Dynamics Observatory (SDO) launched on 2010 Feb. 11, will undoubtedly shed new light on the problem of active region loop heating. The Helioseismic and Magnetic Imager (HMI) onboard SDO will provide a high cadence of vector magnetogram data. These observations will provide detailed temporal information of the photospheric boundary evolution with which to place in context the observations by the Atmospheric Imaging Assembly (AIA) and its EUV observations in various coronal temperatures. The data from this instrument suite will complement the analysis available, as demonstrated here, from the Magnetic Charge Topology and Minimum Coronal Current models for further insights into 
the mechanics of active region heating.

We thank Bish Ishibashi for the helpful comments on this paper. We thank the anonymous referee for his/her comments that improved this paper. This work was supported by NASA grant NNM07AA02C to the Smithsonian Astrophysical Observatory and by the Air Force Office of Scientific Research contract FA9550-06-C-0019 to NorthWest Research Associates. Hinode is a Japanese mission developed and launched by ISAS/JAXA, collaborating with NAOJ as a domestic partner, NASA and STFC (UK) as international partners. Scientific operation of the Hinode mission is conducted by the Hinode science team organized at ISAS/JAXA. This team mainly consists of scientists from institutes in the partner countries. Support for the post-launch operation is provided by JAXA and NAOJ (Japan), STFC (U.K.), NASA, ESA, and NSC (Norway).

\section{A. Appendix}

Figure 11 shows several loops and their ends that produce enhancements in light curves. Using these loops, we estimate the height of the loops that contribute to the light curves (see $\S 4.1)$.

Panel a) shows structures (XL4) that contribute to the light curves in Box 4. The region that contributes to the light curves in Box 4 is a diffuse area and does not show as a single loop, and there are no changes in X-ray and EUV emission. The location of the structure for the Box 4 is not represented in panel b) because the loop is not seen on the TRACE observations. An estimated height of loops using the ends of loops (F4) is represented in Table 4 (see also $§ 4.1)$.

The transient brightenings in the light curve Box 2 and Box 3 are produced by several different loops at different times. Therefore, in panels c)-h), we show the loops that produce enhancements in the light curves of Box 2 and Box 3 at three different times when the transient brightenings are observed by XRT and/or TRACE. In panel c), each loop appearing in Box 2 (XL2) and Box 3 (XL3') that shows enhancements in the light curve in the XRT observation at around 20:00 UT is represented. The ends of loop seen by XRT for Box 2 is marked by F2 in panel c). The loop (TL3') that shows enhancements in the TRACE light curve in Box 3 is a small loop shown in panel d). In panels e) and f), we show the loops that correspond to enhancements at around 22:00 UT. The ends of loop seen by XRT and TRACE for Box 3 are marked with F3 and TF3 in panels e) and f), respectively. In panel e), the loop (XL2') that contributes to the light curve Box 2 at that time is also shown. In panel $\mathrm{g}$ ), the loop (XL2") that shows the enhancement in the light curve for Box 2 at 
around 01:00 UT is shown. At this time, there is no distinct loop in Box 3 in the XRT observation, although there is a small enhancement in the light curve. In panel h), no loops are represented because there is no enhancement in the light curves and brightening in the TRACE observations at this time. The loops appearing in Box 2 and Box 3 at the last three times in Figure 11 look like different loops (see animation movie), though their locations are similar.

We represent the height of loops, L1, XL2, XL3, TL3, and XL4, in Table 4. The distance between the ends of loops, XL3', TL3', XL2', and XL2', are similar or smaller than the loops in Table 4. Therefore, the heights of these loops are lower than the heights of the loops in Table 4.

\section{REFERENCES}

Antiochos, S. K., Karpen, J. T., DeLuca, E. E., Golub, L., \& Hamilton, P. 2003, ApJ, 590, 547

Antolin, P. \& Shibata, K. 2010, ApJ, 712, 494

Aschwanden, M. J., Schrijver, C. J., Winebarger, A. R., \& Warren, H. P. 2003, ApJ, 588, L49

Barnes, G., Longcope, D., \& Leka, K. D. 2005, ApJ, 629, 561

Baum, P. J. \& Bratenahl, A. 1980, Sol. Phys., 67, 245

Berger, T. E., de Pontieu, B., Schrijver, C. J., \& Title, A. M. 1999, ApJ, 519, L97

Beveridge, C. \& Longcope, D. W. 2005, Sol. Phys., 227, 193

Cargill, P. J., Mariska, J. T., \& Antiochos, S. K. 1995, ApJ, 439, 1034

Démoulin, P., Henoux, J. C., \& Mandrini, C. H. 1994, A\&A, 285, 1023

des Jardins, A., Canfield, R., Longcope, D., McLinden, E., \& Dillman, A. 2009, ApJ, 693, 886

Fisher, G. H., Longcope, D. W., Metcalf, T. R., \& Pevtsov, A. A. 1998, ApJ, 508, 885

Fisher, G. H. \& Welsch, B. T. 2008, in Astronomical Society of the Pacific Conference Series, Vol. 383, Subsurface and Atmospheric Influences on Solar Activity, ed. R. Howe, R. W. Komm, K. S. Balasubramaniam, \& G. J. D. Petrie, 373 
Fludra, A. \& Ireland, J. 2008, A\&A, 483, 609

Golub, L. et al. 2007, Sol. Phys., 243, 63

Gorbachev, V. S. \& Somov, B. V. 1988, Sol. Phys., 117, 77

Handy, B. N. et al. 1999, Sol. Phys., 187, 229

Klimchuk, J. A. 2006, Sol. Phys., 234, 41

Kosugi, T. et al. 2007, Sol. Phys., 243, 3

Kumar, N., Kumar, P., \& Singh, S. 2006, A\&A, 453, 1067

Lau, Y.-T. 1993, Sol. Phys., 148, 301

Longcope, D. 1996, Sol. Phys., 169, 91

Longcope, D., Beveridge, C., Qiu, J., Ravindra, B., Barnes, G., \& Dasso, S. 2007, Sol. Phys., 244,45

Longcope, D. W. 2001, Physics of Plasmas, 8, 5277

-. 2005, Living Reviews in Solar Physics, 2, 7

Longcope, D. W., Barnes, G., \& Beveridge, C. 2009, ApJ, 693, 97

Longcope, D. W. \& Beveridge, C. 2007, ApJ, 669, 621

Longcope, D. W. \& Cowley, S. C. 1996, Physics of Plasmas, 3, 2885

Longcope, D. W. \& Klapper, I. 2002, ApJ, 579, 468

Longcope, D. W. \& Magara, T. 2004, ApJ, 608, 1106

Lundquist, L. L., Fisher, G. H., Metcalf, T. R., Leka, K. D., \& McTiernan, J. M. 2008, ApJ, 689,1388

Nagata, S. et al. 2003, ApJ, 590, 1095

Nitta, N. 2000, Sol. Phys., 195, 123

Noglik, J. B., Walsh, R. W., Maclean, R. C., \& Marsh, M. S. 2009, ApJ, 703, 1923

Ofman, L. \& Wang, T. J. 2008, A\&A, 482, L9

Parker, E. N. 1983a, ApJ, 264, 642 
-. 1983b, ApJ, 264, 635

—. 1988, ApJ, 330, 474

Parnell, C. E., Priest, E. R., \& Golub, L. 1994, Sol. Phys., 151, 57

Parnell, C. E., Smith, J. M., Neukirch, T., \& Priest, E. R. 1996, Physics of Plasmas, 3, 759

Plowman, J. E., Kankelborg, C. C., \& Longcope, D. W. 2009, ApJ, 706, 108

Priest, E. R., Foley, C. R., Heyvaerts, J., Arber, T. D., Mackay, D., Culhane, J. L., \& Acton, L. W. 2000, ApJ, 539, 1002

Priest, E. R. \& Forbes, T. G. 1989, Sol. Phys., 119, 211

Priest, E. R., Longcope, D. W., \& Heyvaerts, J. 2005, ApJ, 624, 1057

Rosner, R., Tucker, W. H., \& Vaiana, G. S. 1978, ApJ, 220, 643

Scherrer, P. H. et al. 1995, Sol. Phys., 162, 129

Schmieder, B., Rust, D. M., Georgoulis, M. K., Démoulin, P., \& Bernasconi, P. N. 2004, ApJ, 601, 530

Shimizu, T. et al. 2007, PASJ, 59, 845

Ugarte-Urra, I., Warren, H. P., \& Brooks, D. H. 2009, ApJ, 695, 642

Ugarte-Urra, I., Winebarger, A. R., \& Warren, H. P. 2006, ApJ, 643, 1245

Vaiana, G. S., Krieger, A. S., \& Timothy, A. F. 1973, Sol. Phys., 32, 81

Warren, H. P., Kim, D. M., DeGiorgi, A. M., \& Ugarte-Urra, I. 2009, ArXiv e-prints

Warren, H. P., Ugarte-Urra, I., Brooks, D. H., Cirtain, J. W., Williams, D. R., \& Hara, H. 2007, PASJ, 59, 675

Warren, H. P. \& Winebarger, A. R. 2006, ApJ, 645, 711

Warren, H. P., Winebarger, A. R., \& Brooks, D. H. 2010, ApJ, 711, 228

Warren, H. P., Winebarger, A. R., Mariska, J. T., Doschek, G. A., \& Hara, H. 2008, ApJ, 677,1395

Welsch, B. T., Fisher, G. H., Abbett, W. P., \& Regnier, S. 2004, ApJ, 610, 1148 
Winebarger, A. R. \& Warren, H. P. 2005, ApJ, 626, 543

Yoshida, T., Tsuneta, S., Golub, L., Strong, K., \& Ogawara, Y. 1995, PASJ, 47, L15 
Table 1. Separator properties

\begin{tabular}{cllll}
\hline \hline Separators & Nulls & Length $(\mathrm{Mm})$ & Height $(\mathrm{Mm})$ & \multicolumn{1}{c}{ Domain } \\
\hline$\Psi_{1}$ & A1/B6 & 89 & 27 & $(\mathrm{P} 5-\mathrm{N} 5)$ \\
$\Psi_{2}$ & A2/B11 & 55 & 8 & $-(\mathrm{P} 3-\mathrm{N} 3)^{*}$ \\
$\Psi_{3}$ & A2/B6 & 153 & 50 & $(\mathrm{P} 5-\mathrm{N} 5)+(\mathrm{P} 5-\mathrm{N} 3)$ \\
$\Psi_{4}$ & A3/B8 & 104 & 22 & $-(\mathrm{P} 4-\mathrm{N} 6)^{*}$ \\
$\Psi_{5}$ & A3/B11 & 44 & 11 & $(\mathrm{P} 3-\mathrm{N} 6)$ \\
$\Psi_{6}$ & A4/B11 & 61 & 11 & $(\mathrm{P} 3-\mathrm{N} 6)+(\mathrm{P} 3-\mathrm{N} 1)$ \\
$\Psi_{7}$ & A4/B6 & 160 & 50 & $(\mathrm{P} 5-\mathrm{N} 5)+(\mathrm{P} 5-\mathrm{N} 3)+(\mathrm{P} 5-\mathrm{N} 2)$ \\
\hline
\end{tabular}

*`_' represents an opposite direction in flux flows between flux domain and separator

Table 2. Connectivity matrix in terms of source and separator fluxes

\begin{tabular}{|c|c|c|c|c|c|c|c|c|}
\hline & $\mathrm{P}_{\infty}$ & $\mathrm{P} 1$ & $\mathrm{P} 2$ & P3 & $\mathrm{P} 4$ & $\mathrm{P} 5$ & $\mathrm{P} 6$ & $\mathrm{P} 7$ \\
\hline N1 & $\Phi_{P_{\infty}}$ & $\Phi_{P 1}$ & * & $\Psi_{6}-\Psi_{5}$ & $\Phi_{P 4}+\Psi_{4}$ & $\Phi_{P 5}-\Psi_{7}$ & $\Phi_{P 6}$ & $\Phi_{P 7}$ \\
\hline $\mathrm{N} 2$ & 0 & 0 & $\Phi_{N 2}-\Phi_{P 3}+\Psi_{6}-\Psi_{2}-\Psi_{7}+\Psi_{3}$ & $\Phi_{P 3}-\Psi_{6}+\Psi_{2}$ & 0 & $\Psi_{7}-\Psi_{3}$ & 0 & 0 \\
\hline N3 & 0 & 0 & $\Phi_{N 3}+\Psi_{2}-\Psi_{3}+\Psi_{1}$ & $-\Psi_{2}$ & 0 & $\Psi_{3}-\Psi_{1}$ & 0 & 0 \\
\hline N5 & 0 & 0 & $\Phi_{N 5}-\Psi_{1}$ & 0 & 0 & $\Psi_{1}$ & 0 & 0 \\
\hline N6 & 0 & 0 & $\Phi_{N 6}-\Psi_{5}+\Psi_{4}$ & $\Psi_{5}$ & $-\Psi_{4}$ & 0 & 0 & 0 \\
\hline
\end{tabular}

Note. $-P_{\infty}$ is a positive source "at infinity" introduced to balance the flux from active region. The flux of $P_{\infty}$ is about $10 \%$ of total unsigned flux.

${ }^{*} \Phi_{N 1}-\Phi_{P_{\infty}}-\Phi_{P 1}-\Phi_{P 6}-\Phi_{P 7}-\Psi_{6}+\Psi_{5}-\Phi_{P 4}-\Psi_{4}-\Phi_{P 5}+\Psi_{7}$

Table 3. Domains and separators that influence light curve boxes

\begin{tabular}{lll}
\hline \hline Boxes & \multicolumn{1}{c}{ Flux domains } & Separators \\
\hline Box 1 & P5-(N3, N2, N1), P2-N1, P4-N1, P7-N1, P1-N1 & $\Psi_{1}, \Psi_{3}, \Psi_{7}$ \\
Box 2 & P5-N3, P2-(N3, N2, N1), P4-N1, P7-N1, P1-N1 & $\Psi_{1}, \Psi_{2}, \Psi_{3}, \Psi_{4}, \Psi_{6}, \Psi_{7}$ \\
Box 3 & P3-N3, P2-(N3, N2, N1), P4-N1, P7-N1, P1-N1 & $\Psi_{1}, \Psi_{2}, \Psi_{3}, \Psi_{4}, \Psi_{6}, \Psi_{7}$ \\
Box 4 & P6-N1, P4-N1, P7-N1, P1-N1 & \\
\hline
\end{tabular}

Note. - Bold represents the domain that used to relate separators. 
Table 4. Loops and estimated heights

\begin{tabular}{lcll}
\hline \hline Loops & Time & Height $(\mathrm{Mm})$ & Note \\
\hline L1 & $22: 24 \mathrm{UT}^{*}$ & 50 & Figure 1 \\
XL2 & $20: 07 \mathrm{UT}$ & 40 & Panel c) in Figure 11 \\
XL3 & $22: 07$ UT & 48 & Panel e) in Figure 11 \\
TL3 & $22: 07$ UT & 14 & Panels f) in Figure 11 \\
XL4 & $22: 24$ UT & 46 & Panels a) in Figure 11 \\
\hline
\end{tabular}

Note. - ' ${ }^{*}$ ' represents a time when the XRT observation shows a brightening.
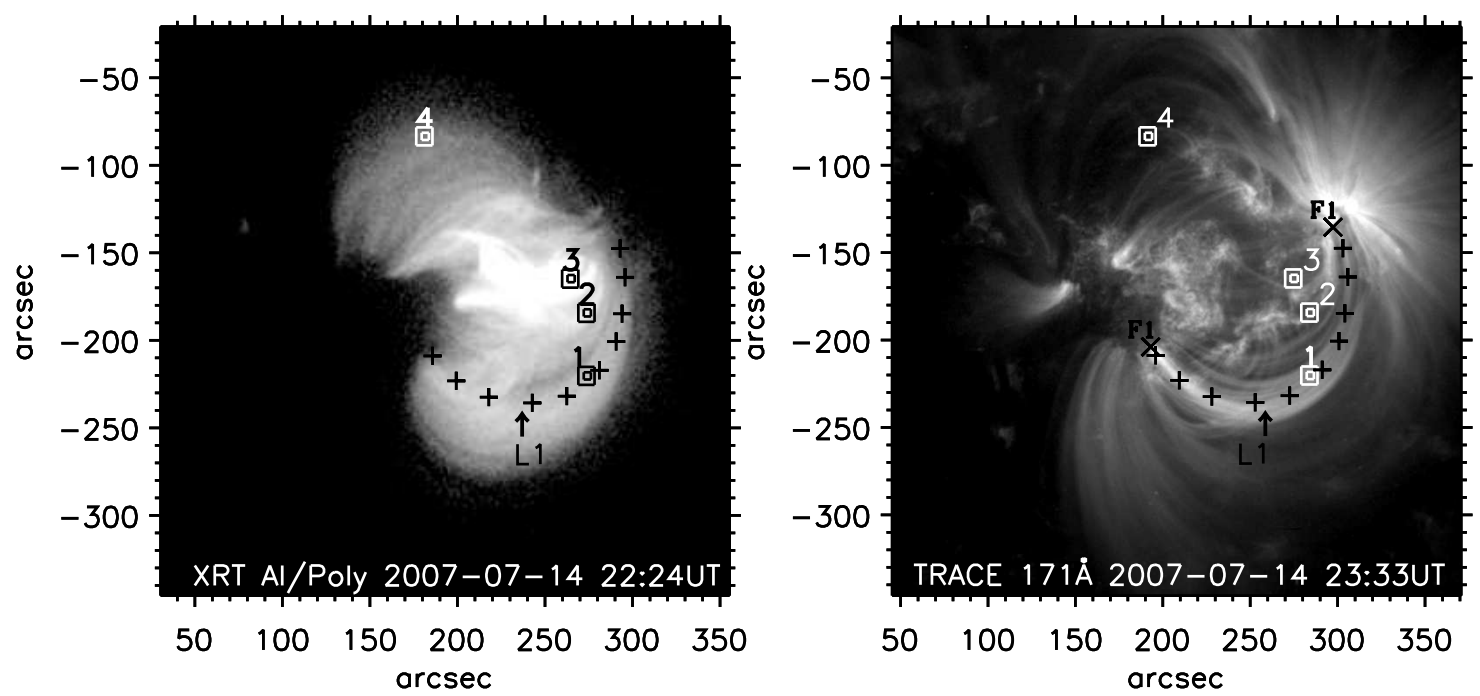

Fig. 1. - The locations of four boxes for light curves (Figure 2) on XRT (left) and TRACE (right) images. Smaller and larger boxes represent the areas of $\sim 5^{\prime \prime} \times 5^{\prime \prime}$ and $\sim 10^{\prime \prime} \times 10^{\prime \prime}$, respectively. A Loop (L1) that produces the enhancement in light curve Box 1 is represented by " + " symbols along the loop. The ends of this loop are represented by F1. This figure is also available as a mpg animation. The movie shows the XRT and TRACE observations for $\sim 6$ hours corresponding to the light curves in Figure 2 , 

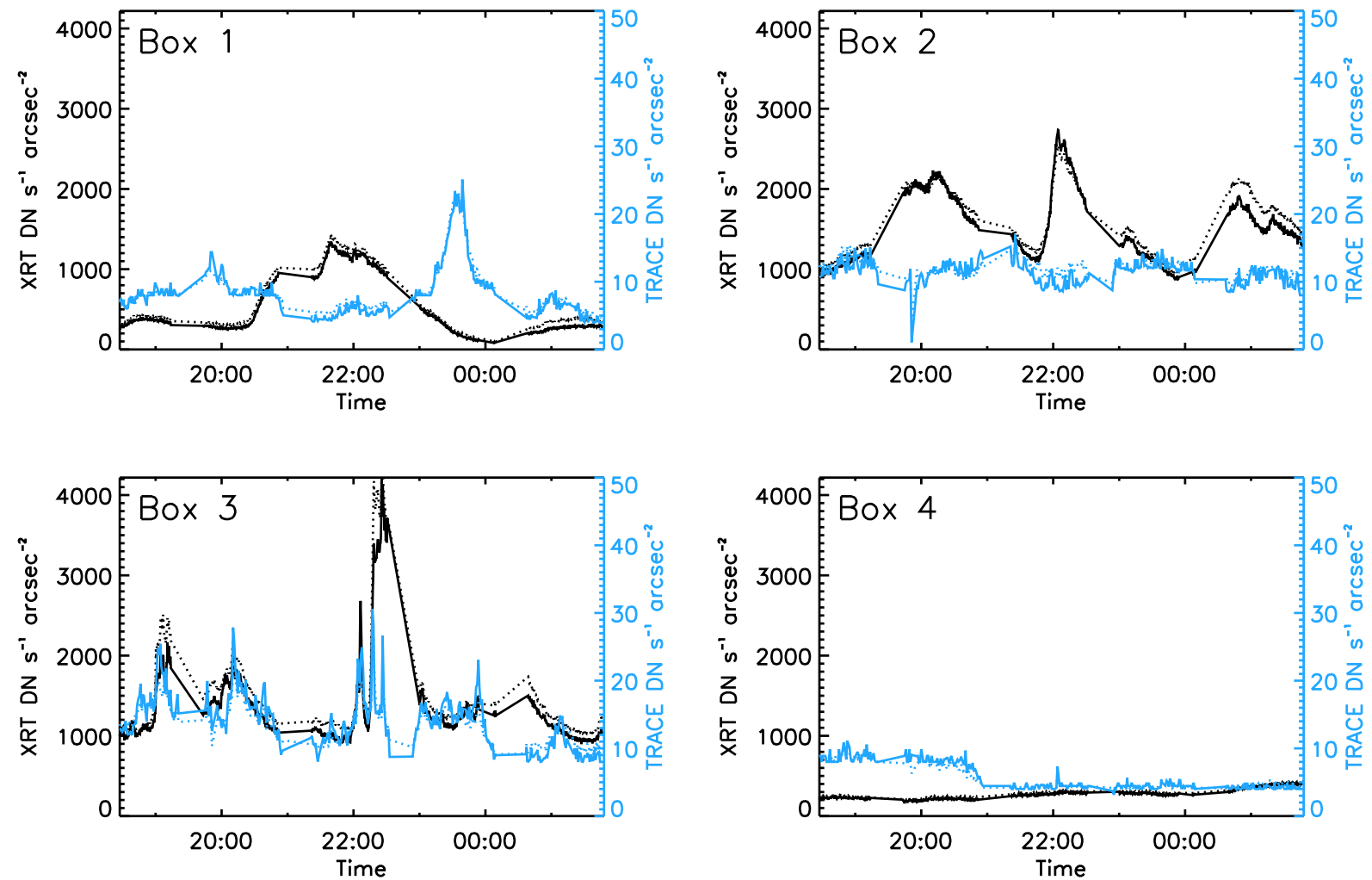

Fig. 2.- Light curves for the four boxes in Figure 1. Black and blue lines (solid: $\sim 5^{\prime \prime} \times 5^{\prime \prime}$, dotted: $\left.10^{\prime \prime} \times 10^{\prime \prime}\right)$ represent XRT and TRACE, respectively. Left and right axes represent the DN s${ }^{-1} \operatorname{arcsec}^{-2}$ for XRT and TRACE, respectively. Left top (Box 1): A brightening in TRACE following a brightening in XRT, indicating a single cooling event. Right top (Box 2): Transient brightenings in XRT and only very small changes in TRACE. Left bottom (Box 3): Transient brightenings in both XRT and TRACE. Right bottom (Box 4): Steady emission in both XRT and TRACE. 

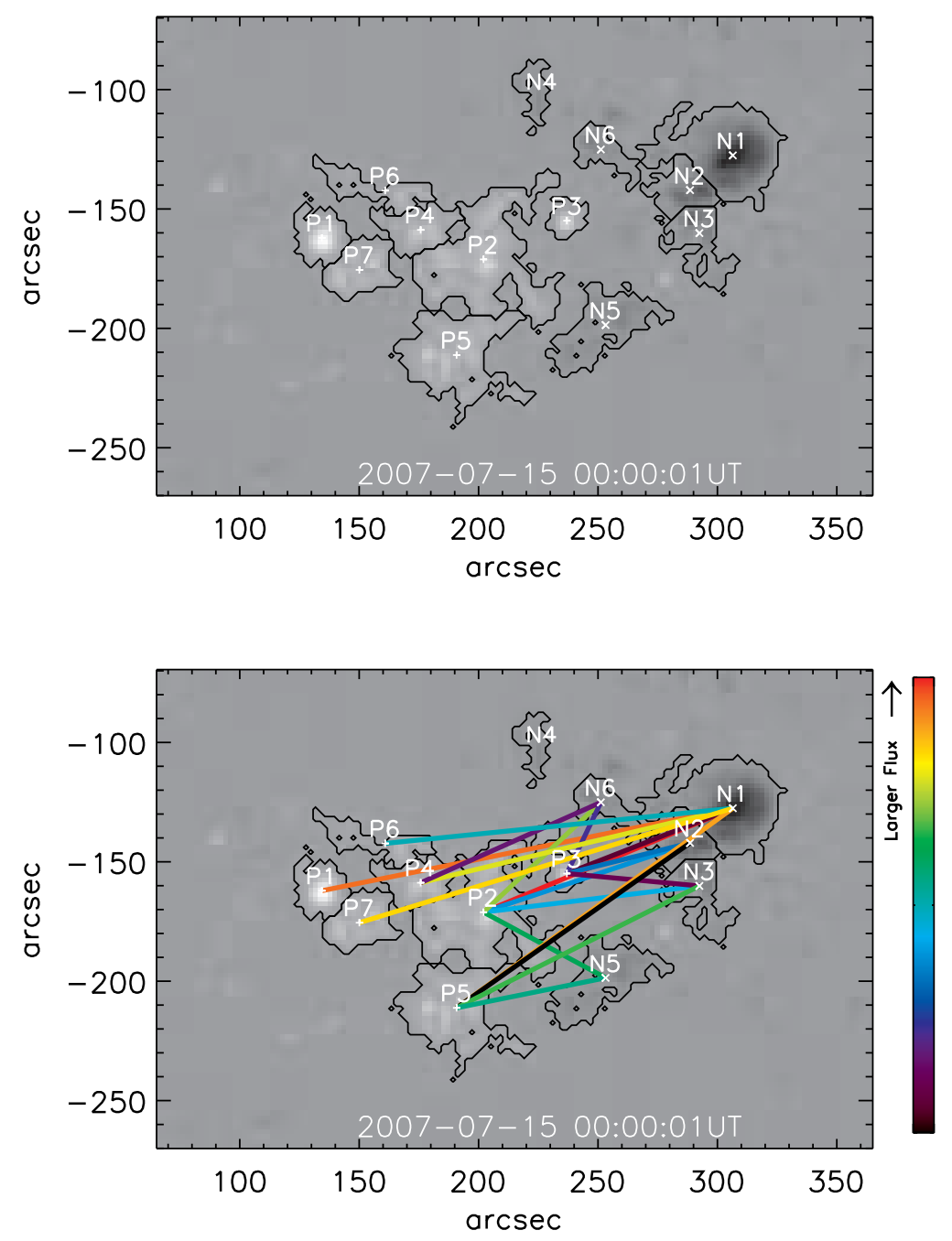

Fig. 3.- Top: Partitioning at 00:00 UT on July 15. Pole locations of positive (P: + ) and negative $(\mathrm{N}: \times)$ sources are represented. Contour represents the partitioning. The background MDI images show only the smoothed field above the threshold used in the partitioning algorithm. Bottom: Colored lines represent domain fluxes among all sources. The contours surrounding individual pixels which are included or excluded from a partition are rendered as small diamonds. 


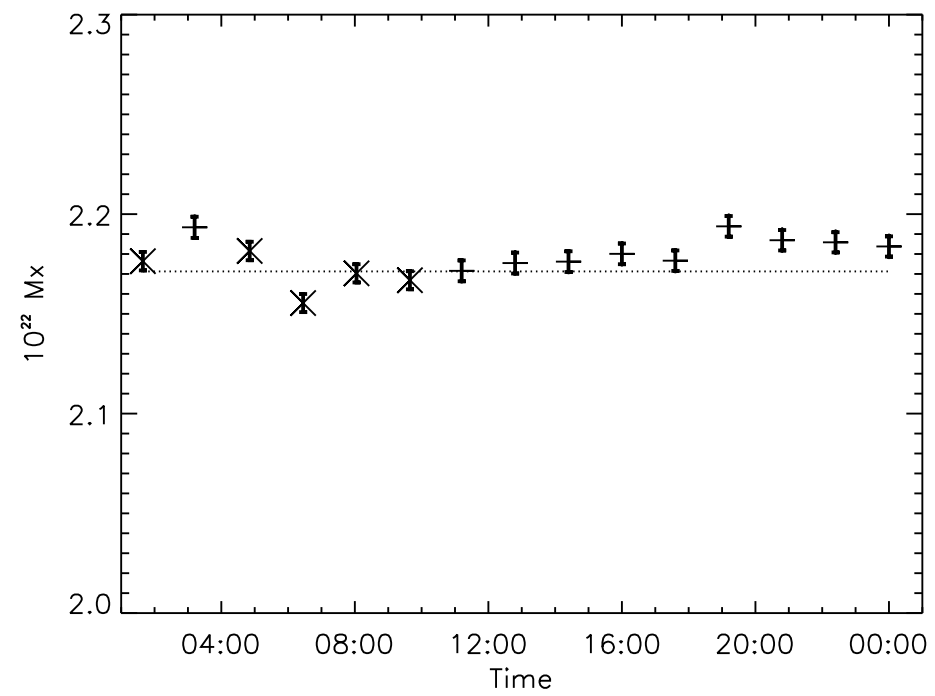

Fig. 4.- Total unsigned source flux for all sources obtained by the time-series partitioning. The integration times of the MDI observations are represented with uncertainties in the total unsigned flux: $300 \mathrm{sec}(\times)$ and $30 \mathrm{sec}(+)$. Dotted line represents the total averaged flux used in this analysis excluding the source flux of $\mathrm{N} 4\left(\Phi_{N 4}=0\right.$, see details in text). Averaged uncertainty for 15 times in the total unsigned flux is $5.0 \times 10^{19} \mathrm{Mx}$. 

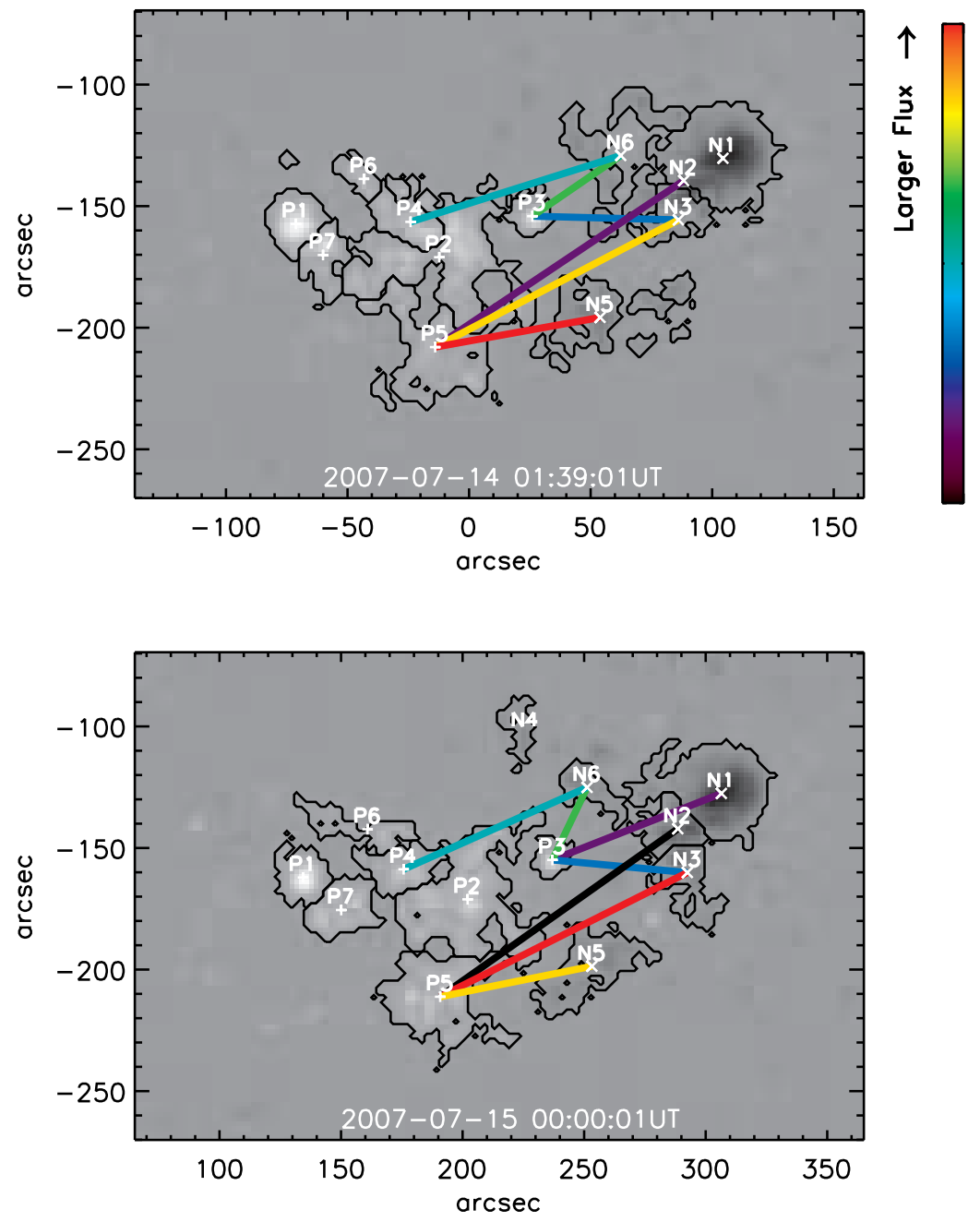

Fig. 5.- Seven domains related to the separators (Table 1) are shown on the top and the bottom panels at 01:39 UT on July 14 and 00:00 UT on July 15, respectively. Colored lines from each positive $(+)$ and negative $(\times)$ source represent the flux in each connection. The scale of color is the same in both top and bottom panels, but this scale is different from the right panel of Figure 3. Footpoint motions and the changes of domain fluxes over the 24 hour period can be seen by comparing the top and the bottom panels. 

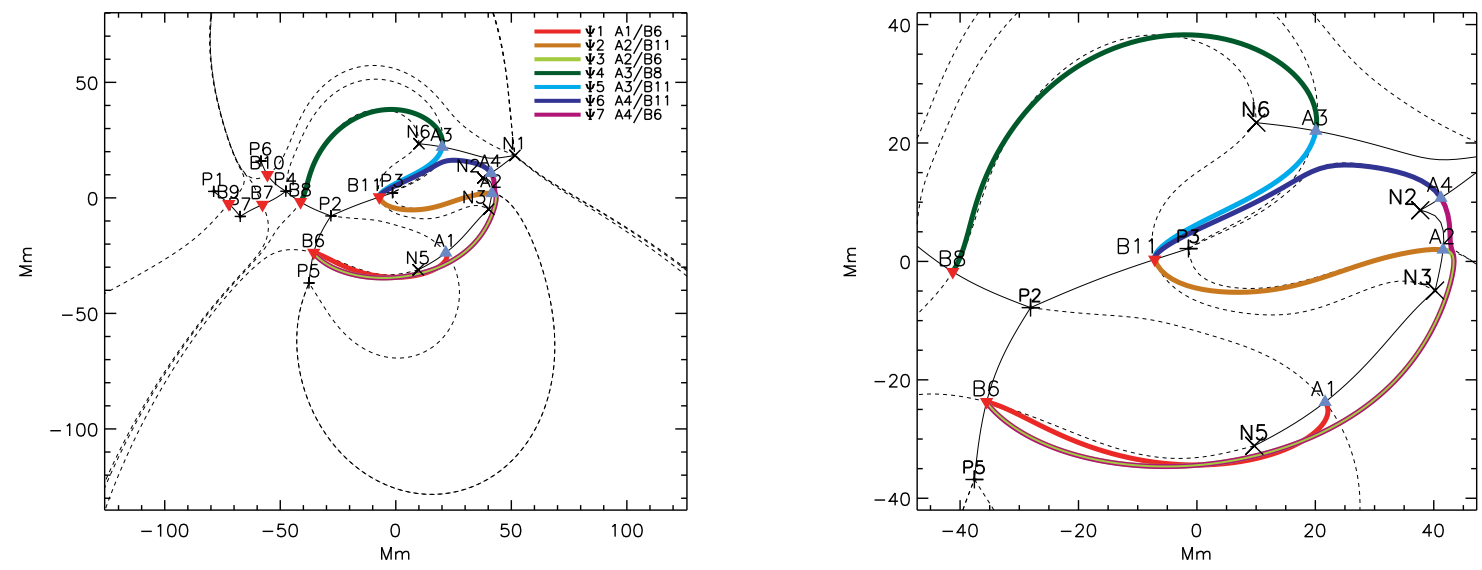

Fig. 6.- Topology of AR 10963 at 00:00 UT on July 15. Right panel shows an enlarged version of the middle of left panel. We show the topology on the tangent plane to explain the domains enclosed by separators in $§ 3.3$. Nulls (B-type: red $\nabla$ and A-type: blue $\triangle$ ) are located between positive and negative sources, respectively. Black dashed and black thin solid lines are fan traces and spines that show where separatrix surfaces intersect the photosphere. Colored lines are the separators: the intersection of separatrix surfaces. $\Psi_{3}$ (A2/B6) is represented by a thinner line than other separators to be visible with $\Psi_{7}(\mathrm{~A} 4 / \mathrm{B} 6)$ because the locations of these two separators are close except between nulls A2 and A4. 


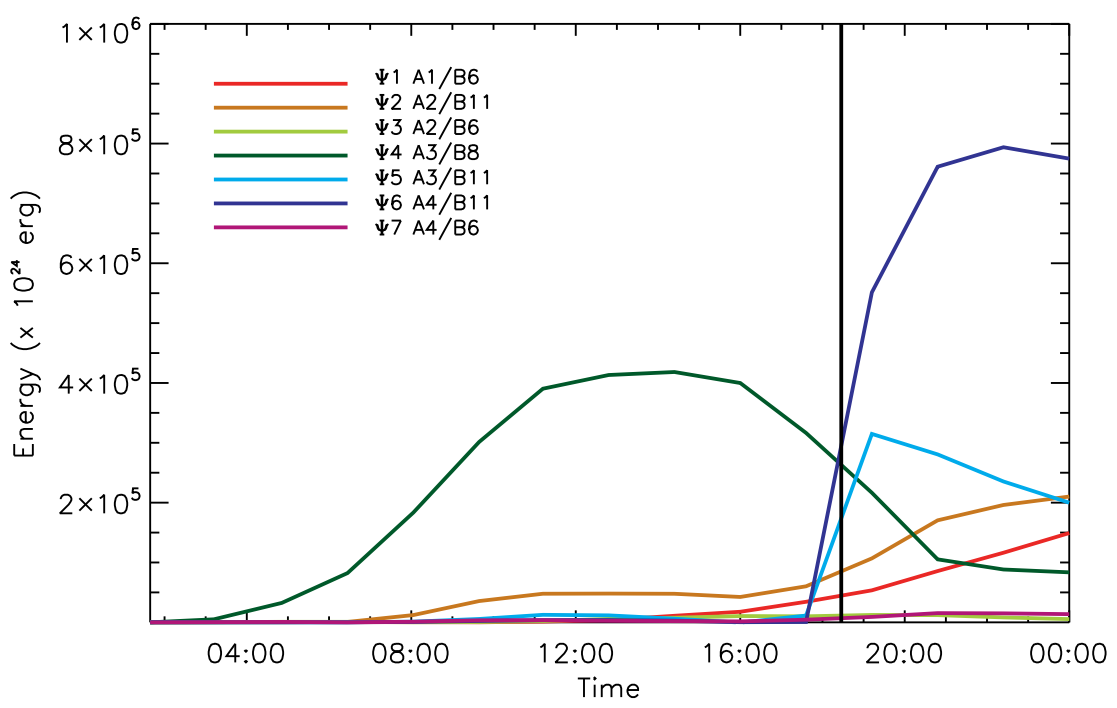

Fig. 7.- Energy buildup from 01:39 UT on July 14 (current=0 at this time) for each separator. Colors are the same as in Figure 6. Black thick line represents the start time of the light curves in Figure 2, $\Psi_{1}$ and $\Psi_{2}$ : A steady increase after $\sim 15: 00 \mathrm{UT} . \Psi_{3}$ and $\Psi_{7}$ : no significant changes in energy from the initial potential state. $\Psi_{4}$ : an increase until $\sim 13: 00 \mathrm{UT}$ and then a decrease. $\Psi_{5}$ : an increase from $\sim 18$ :00 UT and then a decrease. $\Psi_{6}$ : an increase from $\sim 18: 00 \mathrm{UT}$. 

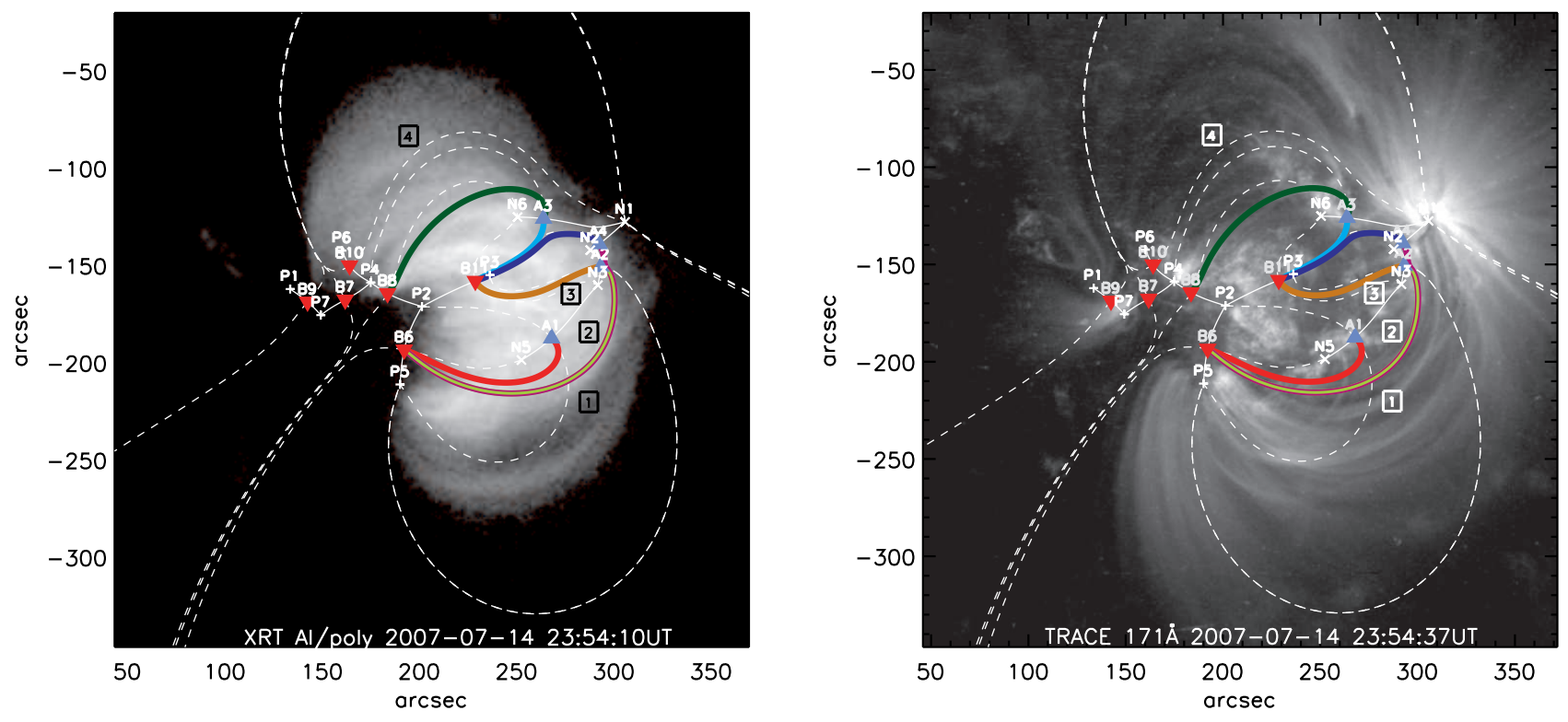

Fig. 8.- Locations of separators on XRT and TRACE observations. The symbols are the same as in Figure 6. Fan traces (white dashed lines), spines (white thin solid lines), and separators (colored lines) are placed on the view from the Earth (image plane). The separator colors are the same as in Figures 6, 7. The locations of separators are slightly shifted comparing with the locations in Figure 6 due to the difference between the image plane and the tangent plane. Light curve boxes are also represented as four boxes. 

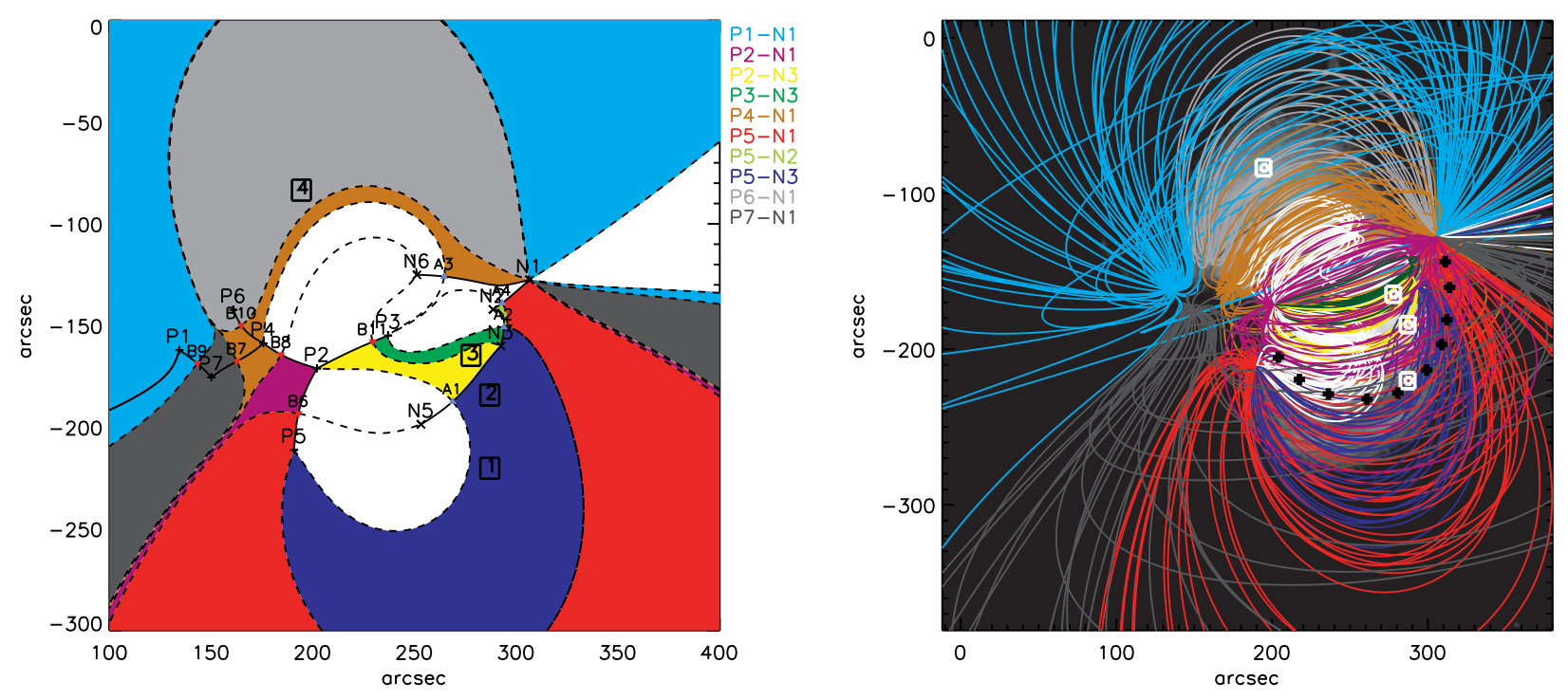

Fig. 9.- Left: Domain footprints on the image plane. Symbols are the same as in Figures 6, 8. The domain footprints are divided by the spines (black solid lines) and fan traces (black dashed lines) on the photosphere. Domains present along the lines of sight for the light curve boxes are shown in color. The positive and negative sources of the domains are labeled on the right side of the figure. Right: Potential field lines of each domain with the same colors as in the left panel. White boxes are the light curve boxes. The loops in Figure 1 are also represented with ${ }^{\prime \prime}+$. 

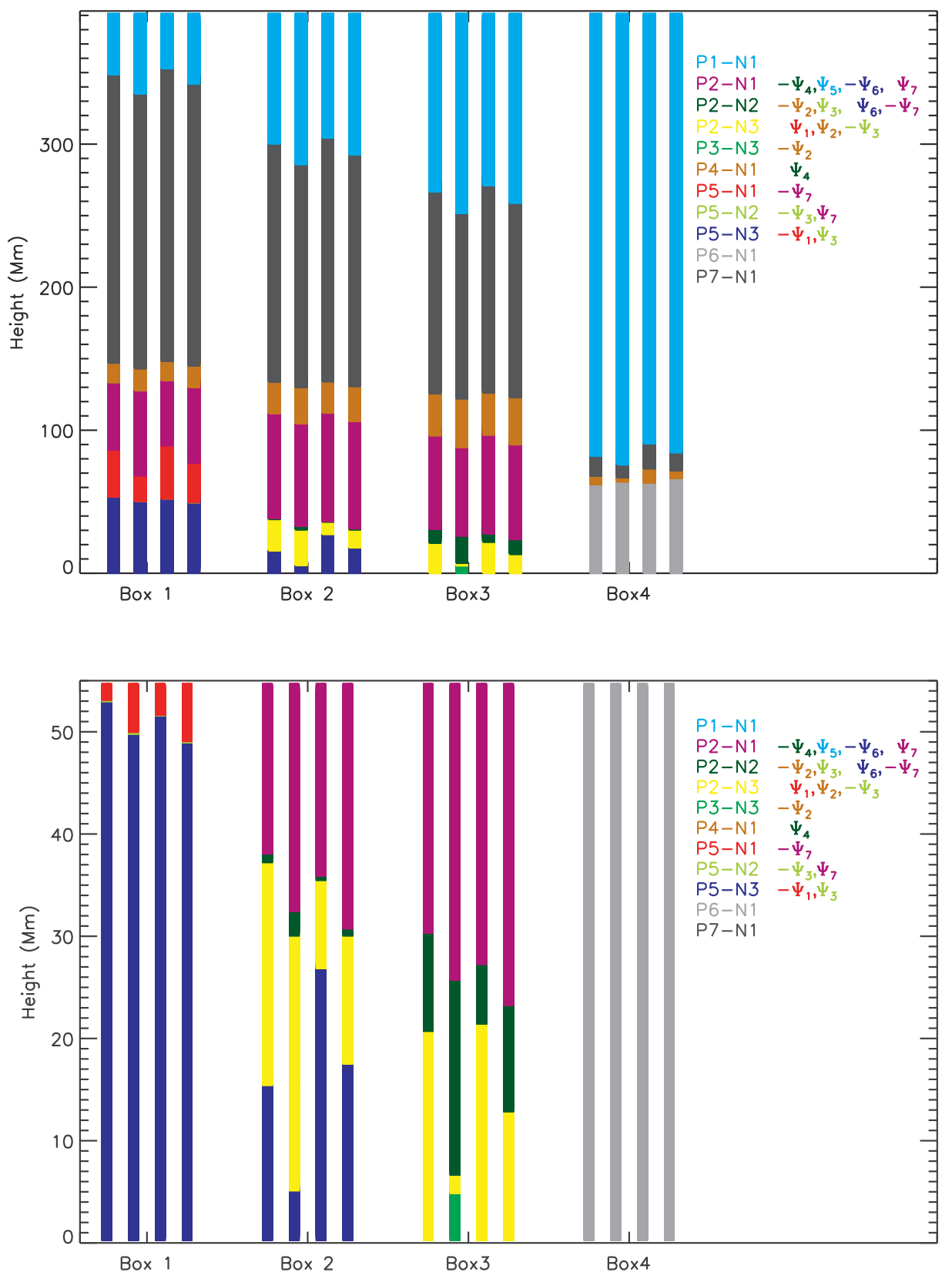

Fig. 10.- Domains along the line of sight through the corona at the locations of the light curve boxes. The top and bottom panels show the domains over a large height range and a small height range, respectively. The domains are shown with the same colors as in Figure9, Each box has four points in the horizontal direction that represent the four corners (SE, NE, SW, NW in order) of $\sim 10^{\prime \prime} \times 10^{\prime \prime}$ square of the boxes. The separators that could affect the domain fluxes are represented on the right of the domains with the same colors as in Figure 8 , 


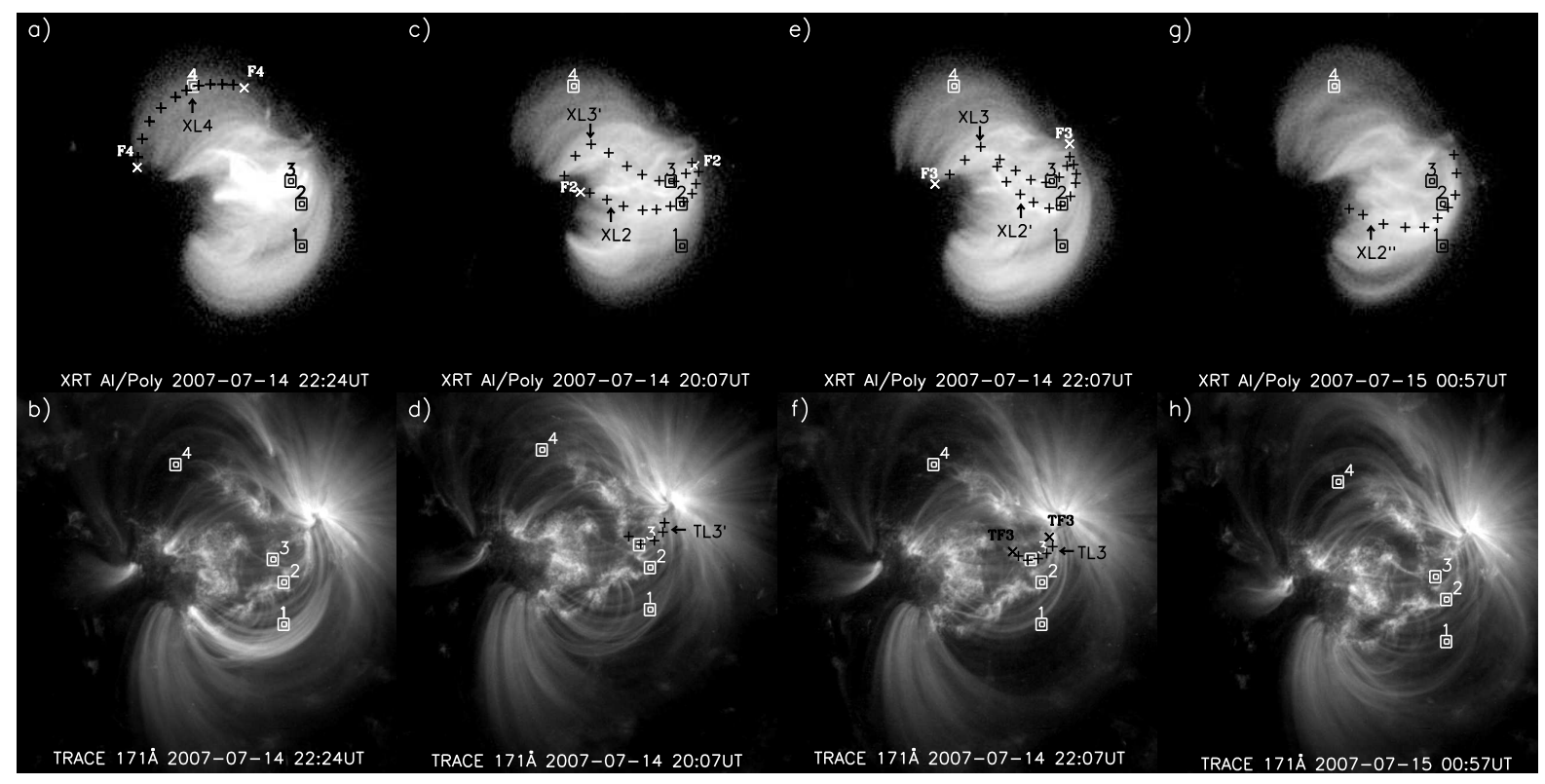

Fig. 11. - Loops that produce the enhancements in light curves are represented by " $+{ }^{\prime \prime}$ symbols along the loop. a) XRT image for loops that contribute to the light curve in Box 4 b) Corresponding TRACE image. c)-h) Loops that produce the enhancements of the light curves Box 2 and Box 3 at three different times in Figure 2. The ends of loops are shown as a symbol " $\times$ " with letters, F2, F3, F4, and TF3 for several loops, which are applied to estimate the heights of the loops (see Table 41). Light curve boxes are also shown as four boxes. 